\title{
ON QUANTUM GAUSSIAN OPTIMIZERS CONJECTURE IN THE CASE $q=p$
}

\author{
A.S. Holevo \\ Steklov Mathematical Institute, RAS
}

Let $\mathcal{H}$ be a separable Hilbert space, $\mathfrak{T}_{p}, 1 \leq p<\infty$, the Schatten class i.e. the Banach space of operators in $\mathcal{H}$ with the finite norm $\|X\|_{p}=\left(\operatorname{Tr}|X|^{p}\right)^{1 / p}$; $\mathfrak{T}_{\infty}$ is the space of compact operators with the operator norm. For $1 \leq p, q \leq \infty$ the norm of a linear map $\Phi: \mathfrak{T}_{p} \rightarrow \mathfrak{T}_{q}$ is defined as

$$
\|\Phi\|_{q \rightarrow p}=\sup _{X \neq 0}\|\Phi[X]\|_{p} /\|X\|_{q}
$$

provided it is finite.

The quantum Gaussian optimizers conjecture, having roots in analysis [5] and in quantum information theory [7], says that if $\Phi$ is Bosonic Gaussian channel (see below) then the supremum is attained on "Gaussian" operators $X$. In the papers [4, 8] the conjecture was proven for $q=1$ and all gauge-covariant (contravariant) Gaussian channels with $s$ degrees of freedom. For arbitrary $p, q>1$ the result was obtained in [2] for rather special class of gauge-covariant channels with $s=1$. Recently the hypothesis was confirmed (in the asymptotic sense) for $q=p$ and gauge-covariant channels with $s \geq 1[3$. The proof is based on a general upper bound from [1] and results from our paper [6] concerning Gaussian channels. In the present note we show that the results of [6] and [9] in fact allow to prove the hypothesis for $q=p$ and all Gaussian channels. Thus the condition of gauge covariance, rather crucial in the case $q=1, s>1$, plays no role in the case $q=p$.

Let $(Z, \Delta)$ be the standard symplectic vector space $(\operatorname{dim} Z=2 s)$ with the nondegenerate skew-symmetric matrix $\Delta$, and let $W(z)=\exp (i R z) ; z \in Z$ be the Weyl system in a Hilbert space $\mathcal{H}$ giving a regular representation for the Canonical Commutation Relations. Here $R$ is the $2 s$-vector row of the canonical observables. Gaussian density operator (d.o.) $\rho$ with mean $m$ and the covariance matrix $\alpha$ satisfying $\alpha \pm \frac{i}{2} \Delta \geq 0$ is defined by the quantum characteristic function

$$
\operatorname{Tr} \rho W(z)=\exp \left(i m^{\top} z-\frac{1}{2} z^{\top} \alpha z\right)
$$

The work was supported by the grant of Russian Scientific Foundation (project No 1421-00162). 
Let $\Phi$ be a general Bosonic Gaussian channel i.e. completely positive map $\mathfrak{T}_{1} \rightarrow \mathfrak{T}_{1}$ such that

$$
\Phi^{*}(W(z))=W(K z) \exp \left(i l^{\top} z-\frac{1}{2} z^{\top} \mu z\right),
$$

where real symmetric matrix $\mu$ satisfies $\mu \geq \pm \frac{i}{2}\left(\Delta-K^{\top} \Delta K\right)$. The channel $\Phi$ transforms a Gaussian d.o. with the covariance matrix $\alpha$ into Gaussian d.o. with the covariance matrix

$$
\alpha^{\prime}=K^{\top} \alpha K+\mu,
$$

see [7] for more detailed description.

THEOREM. Let $\Phi$ be a Bosonic Gaussian channel (3) with invertible matrix $K$, then

$$
\|\Phi\|_{p \rightarrow p}=|\operatorname{det} K|^{1 / p-1}, \quad 1 \leq p \leq \infty,
$$

with the supremum in (1) attained in the limit on a sequence of Gaussian d.o. If $q<p$, then $\Phi$ does not extend to a bounded map $\mathfrak{T}_{p} \rightarrow \mathfrak{T}_{q}$.

Proof. Let us first prove the upper bound

$$
\|\Phi\|_{p \rightarrow p} \leq|\operatorname{det} K|^{1 / p-1} .
$$

The general interpolation bound of [1] implies, as in [3], that for a positive map $\Phi$

$$
\|\Phi\|_{p \rightarrow p} \leq\|\Phi\|_{\infty \rightarrow \infty}^{1-1 / p}\|\Phi\|_{1 \rightarrow 1}^{1 / p}
$$

where $\|\Phi\|_{\infty \rightarrow \infty}=\|\Phi[I]\|$ (in the infinite dimensional case $\Phi[I]$ should be defined as in [6]) and $\|\Phi\|_{1 \rightarrow 1}=1$ in the case of channel.

Now let the matrix $K$ be invertible, then it is proven in [6] that

$$
\Phi[I]=|\operatorname{det} K|^{-1} I,
$$

hence (6) follows.

To prove asymptotic achievability of (6) we follow [6], [9]. We assume $p<\infty$, the case $p=\infty$ can be treated similarly. In [9] it was proven (Eq. (28)) that

$$
\operatorname{Tr} \rho^{p} W(z)=\left[\operatorname{det} f_{p}\left(\operatorname{abs}\left(\Delta^{-1} \alpha_{\beta}\right)\right)\right]^{-\frac{1}{2}} \exp \left[i m^{\top} z-\frac{1}{2} z^{\top} \alpha g_{p}\left(\operatorname{abs}\left(\Delta^{-1} \alpha\right)\right) z\right]
$$

where $f_{p}(d)=(d+1 / 2)^{p}-(d-1 / 2)^{p} \sim p d^{p-1}$ as $d \rightarrow \infty$. For $z=0$ we obtain

$$
\operatorname{Tr} \rho^{p}=\left[\operatorname{det} f_{p}\left(\operatorname{abs}\left(\Delta^{-1} \alpha_{\beta}\right)\right)\right]^{-\frac{1}{2}} .
$$

Let $\rho=\rho_{\beta}$ be the d.o. of the Gibbs state with the Hamiltonian $H=R \epsilon R^{\top}$, where $\epsilon$ is a nondegenerate positive definite matrix (e.g. unit matrix), with the inverse temperature $\beta$. Then $\rho_{\beta}$ is Gaussian with the covariance matrix [6]

$$
2 \Delta^{-1} \alpha_{\beta}=\cot \beta \epsilon \Delta \text {. }
$$


Consider the asymptotic $\beta \rightarrow 0$. Then from (9) $\alpha_{\beta} \sim(2 \beta \epsilon)^{-1}$ and from (4) $\operatorname{det} \alpha_{\beta}^{\prime} \sim|\operatorname{det} K|^{2} \operatorname{det} \alpha_{\beta}$. Hence

$\operatorname{Tr} \rho_{\beta}^{p} \sim p^{-s}\left[\operatorname{det}\left(\operatorname{abs}\left(\Delta^{-1} \alpha_{\beta}\right)\right)\right]^{\frac{1-p}{2}}=p^{-s}\left|\operatorname{det}\left(\Delta^{-1} \alpha_{\beta}\right)\right|^{\frac{1-p}{2}}=p^{-s}\left|\operatorname{det} \alpha_{\beta}\right|^{\frac{1-p}{2}}$.

Similarly

$$
\operatorname{Tr} \Phi\left[\rho_{\beta}\right]^{p} \sim p^{-s}\left|\operatorname{det} \alpha_{\beta}^{\prime}\right|^{\frac{1-p}{2}} \sim p^{-s}\left|(\operatorname{det} K)^{2} \operatorname{det} \alpha_{\beta}\right|^{\frac{1-p}{2}} \sim|\operatorname{det} K|^{1-p} \operatorname{Tr} \rho_{\beta}^{p} .
$$

Hence $\lim _{\beta \rightarrow 0} \frac{\operatorname{Tr} \Phi\left[\rho_{\beta}\right]^{p}}{\operatorname{Tr} \rho_{\beta}^{p}}=|\operatorname{det} K|^{1-p}$ and taking into account (6), equality (5) follows.

The second statement follows from the fact that $\left\|\rho_{\beta}\right\|_{p}$ scales as $\beta^{\frac{s-1}{p}}$ while $\left\|\Phi\left[\rho_{\beta}\right]\right\|_{q}$ as $\beta^{s \frac{q-1}{q}}$ so that the ratio of the norms goes to $\infty$ for $q<p$.

\section{References}

[1] Beigi S. Sandwiched Rényi divergence satisfies data processing inequality. J. Math. Phys. 2013. V. 54. 122202.

[2] De Palma G., Trevisan D., Giovannetti V. One-mode quantum-limited Gaussian channels have Gaussian maximizers. arXiv:1610.09967.

[3] Frank R. L., Lieb E. H. Norms of quantum Gaussian multi-mode channels. J. Math. Phys. 2017. V. 58. 062204.

[4] Giovannetti V., Holevo A.S., Mari A. Majorization and additivity for multimode bosonic Gaussian channels. Theor. Math. Phys. 2015. V. 182. N.2. P. 338-349.

[5] Lieb E. H. Gaussian kernels have only Gaussian maximizers. Invent. Math. 1990. V. 102. P. 179-208.

[6] Holevo A. S. Entropy gain and the Choi-Jamiolkowski correspondence for infinite-dimensional quantum evolutions. Theoret. and Math. Phys. 2011. V. 166. N. 1. P. $123-138$.

[7] Holevo A. S., Quantum systems, channels, information. De Gruyter, Berlin/Boston, 2012.

[8] Holevo A. S. Gaussian optimizers and the additivity problem in quantum information theory. Russian Math. Surveys 2015. V. 70:2, P. 331-367.

[9] Holevo A.S., Sohma M., Hirota O. Error exponents for quantum channels with constrained inputs. Rep. Math. Phys. 2000. V. 46. P. 343-358. 